\title{
Non-Gaussian GARCH option pricing models and their diffusion limits
}

\author{
Alexandru Badescu ${ }^{1}$, Robert J. Elliott ${ }^{2,3,4}$, and Juan-Pablo Ortega ${ }^{5}$
}

\begin{abstract}
This paper investigates the weak convergence of general non-Gaussian GARCH models together with an application to the pricing of European style options determined using an extended Girsanov principle and a conditional Esscher transform as the pricing kernel candidates. Applying these changes of measure to asymmetric GARCH models sampled at increasing frequencies, we obtain two risk neutral families of processes which converge to different bivariate diffusions, which are no longer standard Hull-White stochastic volatility models. Regardless of the innovations used, the GARCH implied diffusion limit based on the Esscher transform can be obtained by applying the minimal martingale measure under the physical measure. However, we further show that for skewed GARCH driving noise, the risk neutral diffusion limit of the extended Girsanov principle exhibits a non-zero market price of volatility risk which is proportional to the market price of the equity risk, where the constant of proportionality depends on the skewness and kurtosis of the underlying distribution. Our theoretical results are further supported by numerical simulations and a calibration exercise to observed market quotes.
\end{abstract}

Keywords: finance, non-Gaussian GARCH models, extended Girsanov principle, conditional Esscher transform, bivariate diffusion limit.

\footnotetext{
${ }^{1}$ Department of Mathematics and Statistics, University of Calgary, Calgary, Canada.

${ }^{2}$ Corresponding author: School of Mathematical Sciences, University of Adelaide, SA 5005, Australia.

${ }^{3}$ Centre for Applied Financial Studies, University of South Australia, Adelaide, Australia.

${ }^{4}$ Haskayne Business School, University of Calgary, Calgary, Canada.

${ }^{5}$ Centre National de la Recherche Scientifique, Laboratoire de Mathématiques de Besançon, Université de Franche-Comté, Besançon, France.
} 


\section{Introduction}

Pricing options based on stochastic volatility (SV) models has been extensively studied in the financial literature. The Generalized Autoregressive Conditionally Heteroskedastic (GARCH) models introduced by Engle (1982) and Bollerslev (1986) have emerged as one of the most popular discrete time alternatives to continuous time bivariate diffusions, not only for their ease of estimation, but also for their performance in capturing the main stylized features of asset returns and option data.

Since markets are incomplete under GARCH models, the choice of the pricing kernel plays an important role. Based on an equilibrium argument, Duan (1995) proposed the local risk neutral valuation relationship (LRNVR) for the pricing of European options under a GARCH model based on Gaussian innovations. More recently, various other GARCH option pricing models using different distributional assumptions on the driving noise have been proposed. For example, Menn and Rachev (2005) uses an $\alpha$-stable family of distributions, Stentoft (2008) a Normal Inverse Gaussian distribution (NIG), Chorro et al. (2011a) a Generalized Hyperbolic, and Badescu et al. (2008) a mixture of Gausians. For further recent advances in the GARCH option pricing literature we refer to the survey paper by Christoffersen et al. (2013b) and references therein. Since Duan's LRNVR requires asset returns to be conditionally Gaussian distributed, different other pricing kernels have been used for option valuation within the GARCH setting: the generalized LRNVR (Duan (1996), Christoffersen et al. (2010), the extended Girsanov principle (EGP) of Elliott and Madan (1998) (e.g. Badescu and Kulperger (2008), Badescu et al. (2008)), the conditional Esscher transform (e.g. Siu et al. (2004), Badescu and Kulperger (2008), Christoffersen et al. (2009)), second-order Esscher transform, variance dependent pricing kernels (Monfort and Pegoraro (2012), Christoffersen et al. (2013a)).

The connection between GARCH type models and diffusion processes has been also investigated in the last decade. Nelson (1990) is the first study on the weak convergence of GARCH models to uncorrelated bivariate diffusions. His results were subsequently extended by Duan (1997) to a more general class of augmented GARCH models. One of the main issues when analyzing the weak limits of GARCH models is the non-uniqueness of the parametric constraints required for convergence which may lead to different limit 
processes. For instance, Corradi (2000) derived a bivariate diffusion limit with a deterministic variance process. Alexander and Lazar (2005) provided a detailed discussion on the impact of time aggregation on the continuous limit and derived a convergence result for weak GARCH models. For a survey on continuous time limits of GARCH and volatility models we refer to Lindner (2009). Despite the growing literature on such convergence results, there are not many studies which investigate the interplay between GARCH and their diffusion limits with applications to pricing and hedging derivatives. For this purpose, one has to analyze the weak convergence under both the physical and risk neutral measures under the same parametric restrictions. An initial study in this sense has been provided by Duan (1996) who derived the minimal martingale measure as the weak limit of his LRNVR for a Gaussian GARCH model. This result has been numerically tested by, amongst others, Stentoft (2011) for European and American style options. Heston and Nandi (2000) derives the continuous limit of an affine Gaussian GARCH model based on different parametric constraints. Duan et al. (2009) studied the convergence speed of European options under the normally distributed GARCH driving innovations, while Fornari and Mele (1997) investigated the weak convergence of a risk neutral non-linear ARCH model. The only paper that, to our knowledge, studied this joint convergence outside a Gaussian framework is Duan et al. (2006), who proposed a GARCH-jump option pricing model based on a Poisson random sum of Gaussian random variables. The pricing kernel used in their paper can be viewed as a special case of a conditional Esscher transform.

Motivated by the well-documented empirical features exhibited by financial data, this paper aims to bridge the gap between GARCH and diffusions based option pricing techniques under a general non-Gaussian framework. More specifically, we derive the continuous time limits of a discretized asymmetric GARCH option pricing model driven by an innovation process with a finite moment generating function and we numerically test the convergence performance of European option prices under a specific distributional assumption. Using parametric constraints for the underlying discrete-time model in the spirit of Nelson (1990), we obtain as its continuous time limit a bivariate diffusion with a coefficient of the variance process that depends on the skewness and kurtosis of the asset 
returns.

Next, we adapt the construction of the extended Girsanov principle and the conditional Esscher transform to our discretized GARCH model, and derive the risk neutralized dynamics resulting from the measure change. Using the same parametric constraints under which the physical convergence has been established, we derive the weak limit of the risk neutral process under the two stochastic discount factors. The resulting limits are two bivariate diffusions driven by two correlated Brownian motions which differ only through the drift term which no longer has a Hull-White (1987) structure. In the Esscher case, regardless of the innovation distribution, the GARCH diffusion limit coincides to the risk neutral process obtained by applying the minimal martingale measure (MMM) to the continuous time process under the physical measure; this corresponds to a zero market price of volatility risk. However, in the extended Girsanov case, we obtain a risk neutral GARCH limit which exhibits a non-zero market price of variance risk when the distribution of the GARCH innovations are skewed. The two continuous time limits coincide if returns are modeled with symmetric densities. Moreover, when they are conditionally Gaussian distributed we recover the limiting process of Duan(1995) obtained via the LRNVR. From the points stated above, our results can be viewed as extensions of the LRNVR to non-Gaussian GARCH models.

Our theoretical results are further supported by two numerical experiments. First, we compare European option prices based on NIG-GARCH processes sampled at daily frequency and their continuous time limits. In the EGP case, we also analyze numerically the convergence of the discrete model by considering different sampling frequencies. Our results suggest that there are no significant differences between option prices based on the discrete and continuous counterparts for short-dated options, but the differences become more pronounced for larger maturities. In most cases, the pricing errors also converge smoothly to zero when the time interval between observations is small. Secondly, we analyze the effect of the non-zero market price of volatility risk by comparing the performance of the two GARCH diffusion limits calibrated to observed market prices on a particular day. We find that the EGP stochastic discount factor outperforms the Esscher transform for both GARCH option pricing models and their continuous time 
diffusion limits.

The paper is organized as follows. Section 2 introduces the non-Gaussian asymmetric GARCH model that we are interested in as well as its diffusion limit. The martingale measures and the main convergence result for the risk-neutralized models are provided in Section 3. In Section 4 we perform the numerical experiments. Section 5 concludes the paper.

Acknowledgements: AB thanks the Natural Sciences and Engineering Research Council of Canada (NSERC) and the Montreal Institute of Financial Mathematics (IFM2) for their support. RE thanks the Australian Research Council (ARC) and NSERC. We thank Lyudmila Grigoryeva for her help in the construction of the computer codes that were used in the numerical section of the paper. We also acknowledge comments from John Maheu, Lars Stentoft, the editor, and two anonymous referees that significantly improved the contents of the paper.

\section{Non-Gaussian GARCH and its diffusion limit}

Consider a $n$-indexed discrete time financial market with the set of equally spaced trading dates $\mathcal{T}^{(n)}=\{l \mid l=k / n, k=0,1 \ldots, n T\}$. The length of each subinterval is denoted by $\tau=1 / n$. Real-world dynamics are defined on a filtered probability space $\left(\Omega^{(n)}, \mathcal{F}^{(n)},\left\{\mathcal{F}_{l}^{(n)}\right\}_{l \in \mathcal{T}^{(n)}}, P^{(n)}\right)$. We assume that the logarithm of the risky asset price process, denoted by $\left\{Y_{l}^{(n)}\right\}_{l \in \mathcal{T}^{(n)}}=\left\{\log S_{l}^{(n)}\right\}_{l \in \mathcal{T}^{(n)}}$, has the following stochastic volatility structure for any $k=0,1 \ldots, n T$ :

$$
\begin{aligned}
Y_{k \tau}^{(n)} & =Y_{(k-1) \tau}^{(n)}+\left(r+\lambda^{(\epsilon)} \sqrt{h_{k \tau}^{(n)}}-\kappa_{k \tau}^{(n)}\left(\sqrt{h_{k \tau}^{(n)}}\right)\right) \tau+\sqrt{\tau h_{k \tau}^{(n)}} \epsilon_{k \tau}^{(n)}, \\
h_{k \tau}^{(n)} & =\alpha_{0}(\tau)+\alpha_{1}(\tau) h_{(k-1) \tau}^{(n)}\left(\epsilon_{(k-1) \tau}^{(n)}-\gamma(\tau)\right)^{2}+\beta_{1}(\tau) h_{(k-1) \tau}^{(n)} .
\end{aligned}
$$

We assume that $\mathcal{F}_{k \tau}^{(n)}$ is the $\sigma$-field generated by the historical asset prices, $\mathcal{F}_{k \tau}^{(n)}=$ $\sigma\left(Y_{0}^{(n)}, Y_{1}^{(n)}, \ldots, Y_{k \tau}^{(n)}\right)$ for any $k=0, \ldots, n T ;\left\{\epsilon_{k \tau}^{(n)}\right\}_{k=0, \ldots, n T}$ is a sequence of $\mathcal{F}_{(k-1) \tau}^{(n)}$ - conditionally iid random variables with zero mean and unit variance distribution D, $\epsilon_{k \tau}^{(n)} \mid \mathcal{F}_{(k-1) \tau}^{(n)} \sim \mathbf{D}(0,1)$. We denote by $f_{\epsilon_{k \tau}}^{(n)}(\cdot)$ the conditional probability density function and we let $\kappa_{\epsilon_{k \tau}}^{(n)}(\cdot)$ be the cumulant generating function of the driving noise under the 
physical measure $P^{(n)}$ which is assumed to be smooth and bounded:

$$
\kappa_{\epsilon_{k \tau}}^{(n)}(u):=\ln \mathrm{E}^{P^{(n)}}\left[\exp \left(u \epsilon_{k \tau}^{(n)}\right) \mid \mathcal{F}_{(k-1) \tau}^{(n)}\right]
$$

Finally, the innovations' $j^{\text {th }}$ raw moments are denoted by

$$
M_{j}=\mathrm{E}^{P^{(n)}}\left[\left(\epsilon_{k \tau}^{(n)}\right)^{j} \mid \mathcal{F}_{(k-1) \tau}^{(n)}\right] .
$$

and are assumed to be finite and not dependent on $\tau$. The conditional mean return equation (2.1) has an affine form in the conditional volatility where $r$ represent the instantaneous constant risk free rate of return and the parameter $\lambda^{(\epsilon)}$ quantifies the market price of $\epsilon$ risk. The conditional variance process $\left\{h_{k \tau}^{(n)}\right\}_{0 \leq k \leq n T}$ has a GARCHtype structure with time varying parameters $\alpha_{0}(\tau), \alpha_{1}(\tau), \beta_{1}(\tau)$, and $\gamma(\tau)$ satisfying the standard non-negativity and covariance stationarity properties. Note that in the special case $\tau=1$, the model (2.1)-(2.2) reduces to a general asymmetric $\operatorname{NGARCH}(1,1)$ model.

The right continuous with left limit (cadlag) extension of the proposed discrete time process is defined by:

$$
\left\{Y_{t}^{(n)}, h_{t}^{(n)}\right\}_{k \tau \leq t<(k+1) \tau}:=\left\{Y_{k \tau}^{(n)}, h_{k \tau}^{(n)}\right\}, \quad k=0, \ldots, n T
$$

Similarly, we define the continuous filtration, $\left\{\mathcal{F}_{t}^{(n)}\right\}_{k \tau \leq t<(k+1) \tau}:=\mathcal{F}_{k \tau}^{(n)}, k=0, \ldots, n T$, and we denote by $\mathcal{F}_{t}^{(n), h_{t}}:=\mathcal{F}_{t}^{(n)} \bigcup\left\{h_{t}^{(n)}=h_{t}\right\}$. Following parametric constraints in the spirit of Nelson (1990) and Duan (1997), we characterize the continuous time weak limit of our model in the proposition below.

Proposition 2.1 Assume the following parameter conditions hold:

$\lim _{\tau \rightarrow 0} \frac{\alpha_{0}(\tau)}{\tau}=\omega_{0}, \quad \lim _{\tau \rightarrow 0} \frac{\alpha_{1}(\tau)\left(1+\gamma^{2}(\tau)\right)+\beta_{1}(\tau)-1}{\tau}=-\omega_{1}, \quad \lim _{\tau \rightarrow 0} \frac{\alpha_{1}^{2}(\tau)}{\tau}=\omega_{2}, \quad \lim _{\tau \rightarrow 0} \gamma(\tau)=\omega_{3}$.

Then, as $\tau$ approaches zero, the process $\left\{Y_{t}^{(n)}, h_{t}^{(n)}\right\}$ converges weakly to a bivariate diffusion $\left(Y_{t}, \sigma_{t}^{2}\right)$ which satisfies the following stochastic differential equation:

$$
\begin{aligned}
& d Y_{t}=\left(r+\lambda^{(\epsilon)} \sqrt{h_{t}}-\kappa_{\epsilon_{t}}\left(\sqrt{h_{t}}\right)\right) d t+\sqrt{h_{t}} d B_{t}^{(1)} \\
& d h_{t}=\left(\omega_{0}-\omega_{1} h_{t}\right) d t+\sqrt{\omega_{2}}\left(M_{3}-2 \omega_{3}\right) h_{t} d B_{t}^{(1)}+\sqrt{\omega_{2}} \sqrt{M_{4}-M_{3}^{2}-1} h_{t} d B_{t}^{(2)}
\end{aligned}
$$

Here, $B_{t}^{(1)}$ and $B_{t}^{(2)}$ are two independent Brownian motions on $\left(\Omega, \mathcal{F},\left\{\mathcal{F}_{t}\right\}_{t \in[0, \ldots, T]}, P\right)$. 
Note that in order to save space, we omit the presentation of the standard technical moment conditions required for the weak convergence of Markov processes (e.g. see Nelson (1990) for details). In the case of Gaussian innovations (i.e. $\kappa_{\epsilon_{t}}\left(\sqrt{h_{t}}\right)=\frac{1}{2} h_{t}, M_{3}=0$ and $\left.M_{4}=3\right)$, the bivariate diffusion from (2.4)-(2.5) coincides with the standard asymmetric GARCH diffusion limit of Duan (1997). The use of a non-Gaussian distribution for the underlying discrete process does not alter the Hull-White structure of the variance equation. However, when compared to the standard Gaussian GARCH limit of Duan (1997), the diffusion coefficient of the stochastic volatility dynamics incorporates the skewness and the kurtosis of the distribution. Throughout the paper, we refer to the model (2.4)-(2.5) as the GARCH diffusion model under $P$.

\section{Convergence of risk neutralized processes}

There are different approaches to investigating the weak convergence of option prices based on discrete time models to their continuous time limits. For instance, once the convergence of the underlying models under the physical measure has been established, a sufficient condition for the convergence of the option prices is provided by the weak convergence of the Radon-Nikodym processes associated to the martingale measures defined in both discrete and continuous time. This methodology has been used in a variety of cases where the valuation is performed using the minimal martingale measure (e.g. see Prigent (2002) and the references therein). The standard approach in the GARCH literature is based on showing the weak convergence between the two risk neutralized processes (e.g. see Duan (1996) and Duan (2006)). In this paper, we use this prescription by analyzing the convergence of risk neutralized GARCH processes based on the extended Girsanov principle and the conditional Esscher transform.

We first describe the construction of the EGP. The main idea is to find a change of

measure such that, the discounted asset prices, denoted by $\widetilde{S}_{k \tau}^{(n)}$ for any $k=0, \ldots, n T$, follow the distribution of their martingale component in the multiplicative Doob decomposition given by:

$$
\widetilde{S}_{k \tau}^{(n)}=\widetilde{S}_{0}^{(n)} A_{k \tau}^{(n)} M_{k \tau}^{(n)}
$$


Here, $A_{k \tau}^{(n)}$ is a $\mathcal{F}_{k \tau}^{(n)}$-predictable process with the following unique representation:

$$
A_{k \tau}^{(n)}=\prod_{l=1}^{k} \mathrm{E}^{P^{(n)}}\left[\frac{\widetilde{S}_{l \tau}^{(n)}}{\widetilde{S}_{(l-1) \tau}^{(n)}} \mid \mathcal{F}_{(l-1) \tau}^{(n)}\right]
$$

The process $M_{k \tau}^{(n)}$ is a martingale under $P^{(n)}$, so that $W_{k \tau}^{(n)}:=M_{k \tau}^{(n)} / M_{(k-1) \tau}^{(n)}$ is a mean one $\mathcal{F}_{k \tau}^{(n)}$ martingale. We denote by $\eta_{k \tau}^{(n)}=\lambda^{(\epsilon)} \sqrt{h_{k \tau}^{(n)}} \tau$ the one period excess discounted return process. The Radon-Nikodym process $Z_{k \tau}^{(n)}$ corresponding to the EGP is defined via the conditional probability density function $g_{W_{k \tau}}^{(n)}(\cdot)$ of $W_{k \tau}^{(n)}$ through the expression:

$$
Z_{k \tau}^{(n)}:=\left.\frac{d Q_{e g p}^{(n)}}{d P^{(n)}}\right|_{\mathcal{F}_{k \tau}^{(n)}}=\prod_{l=1}^{k} \frac{g_{W_{l \tau}}^{(n)}\left(\frac{\widetilde{S}_{l \tau}^{(n)}}{\widetilde{S}_{(l-1) \tau}^{(n)}}\right) e^{\eta_{l \tau}^{(n)}}}{g_{W_{l \tau}}^{(n)}\left(e^{-\eta_{l \tau}^{(n)}} \frac{\widetilde{S}_{l \tau}^{(n)}}{\widetilde{S}_{(l-1) \tau}^{(n)}}\right)} .
$$

The fact that $\left\{Z_{k \tau}^{(n)}\right\}_{k=0, \ldots, n T}$ is a martingale under $P^{(n)}$ and that the new measure $Q_{e g p}^{(n)}$ defined by (3.1) is an equivalent martingale measure with respect to $P^{(n)}$ follows from Theorem 3.1 in Elliott and Madan (1998). In the following proposition, we characterize the risk neutral specification associated to $\left\{Y_{k \tau}^{(n)}, h_{k \tau}^{(n)}\right\}_{k=0, \ldots, n T}$ in (2.1)-(2.2) with respect to the measure that we just introduced.

Proposition 3.1 The risk neutral dynamics of the process $\left\{Y_{k \tau}^{(n)}, h_{k \tau}^{(n)}\right\}_{k=0, \ldots, n T}$ introduced in (2.1)-(2.2) with respect to the extended Girsanov measure $Q_{\text {egp }}^{(n)}$ is given by:

$$
\begin{aligned}
Y_{k \tau}^{(n)} & =Y_{(k-1) \tau}^{(n)}+\left(r-\frac{1}{\tau} \kappa_{k \tau}^{(n)}\left(\sqrt{\tau h_{k \tau}^{(n)}}\right)\right) \tau+\sqrt{\tau h_{k \tau}^{(n)}} \epsilon_{k \tau}^{*(n)} \\
h_{k \tau}^{(n)} & =\alpha_{0}(\tau)+\alpha_{1}(\tau) h_{(k-1) \tau}^{(n)}\left(\epsilon_{(k-1) \tau}^{*(n)}-\sqrt{\tau} \varrho_{(k-1) \tau}^{(n)}-\gamma(\tau)\right)^{2}+\beta_{1}(\tau) h_{(k-1) \tau}^{(n)}
\end{aligned}
$$

Here, the innovation process $\left\{\epsilon_{k \tau}^{*(n)}\right\}_{k=0, \ldots, n T}$ is a sequence of $\mathcal{F}_{(k-1) \tau}^{(n)}$-conditionally uncorrelated zero mean and unit variance $\mathbf{D}$-distributed random variables under $Q_{e g p}^{(n)}, \epsilon_{k \tau}^{*(n)} \mid \mathcal{F}_{(k-1) \tau}^{(n)} \sim$ $\mathbf{D}(0,1)$, related to the original innovations via the expression:

$$
\epsilon_{k \tau}^{*(n)}=\epsilon_{k \tau}^{(n)}+\sqrt{\tau} \varrho_{k \tau}^{(n)}, \quad k=0, \ldots, n T
$$

where $\varrho_{k \tau}^{(n)}$ is given by:

$$
\varrho_{k \tau}^{(n)}=\lambda^{(\epsilon)}+\frac{\frac{1}{\tau} \kappa_{\epsilon_{k \tau}}^{(n)}\left(\sqrt{\tau h_{k \tau}^{(n)}}\right)-\kappa_{\epsilon_{k \tau}}^{(n)}\left(\sqrt{h_{k \tau}^{(n)}}\right)}{\sqrt{h_{k \tau}^{(n)}}} .
$$


We notice that $\varrho^{(n)}=\left\{\varrho_{k \tau}^{(n)}\right\}_{k=0, \ldots, n T}$ is an $\mathcal{F}_{k \tau}^{(n)}$-predictable process under $P^{(n)}$ which depends on the conditional volatility of the driving noise through its cumulant generating function; the quantity $\sqrt{\tau} \varrho_{k \tau}^{(n)}$ represents the mean adjustment of the asset return innovation such that the discounted asset price becomes a martingale after the change of measure.

Next, we derive the risk neutral dynamics of the asset returns under the conditional Esscher transform. Its Radon-Nikodym derivative is given by:

$$
Z_{k \tau}^{(n)}:=\left.\frac{d Q_{e s s}^{(n)}}{d P^{(n)}}\right|_{\mathcal{F}_{k \tau}^{(n)}}=\prod_{l=1}^{k} \exp \left(-\sqrt{\tau} \theta_{l \tau}^{(n)} \epsilon_{l \tau}^{(n)}-\kappa_{\epsilon_{l \tau}}^{(n)}\left(-\sqrt{\tau} \theta_{l \tau}^{(n)}\right)\right)
$$

The Esscher coefficient $\theta^{(n)}=\left\{\theta_{k \tau}^{(n)}\right\}_{k=0, \ldots, n T}$ is a $\mathcal{F}^{(n)}$ predictable process satisfying:

$$
\lambda^{(\epsilon)} \sqrt{h_{k \tau}^{(n)}}-\kappa_{\epsilon_{k \tau}}^{(n)}\left(\sqrt{h_{k \tau}^{(n)}}\right)+\frac{1}{\tau} \kappa_{\epsilon_{k \tau}}^{(n)}\left(\sqrt{\tau}\left(\sqrt{h_{k \tau}^{(n)}}-\theta_{k \tau}^{(n)}\right)\right)-\frac{1}{\tau} \kappa_{\epsilon_{k \tau}}^{(n)}\left(-\sqrt{\tau} \theta_{k \tau}^{(n)}\right)=0
$$

Equation (3.7) ensures the discounted price process is a martingale under $Q_{\text {ess }}^{(n)}$.

Proposition 3.2 The risk neutral dynamics of the process $\left\{Y_{k \tau}^{(n)}, h_{k \tau}^{(n)}\right\}_{k=0, \ldots, n T}$ introduced in (2.1)-(2.2) with respect to the conditional Esscher transform $Q_{\text {ess }}^{(n)}$ is given by:

$$
\begin{aligned}
Y_{k \tau}^{(n)} & =Y_{(k-1) \tau}^{(n)}+\left(r+\frac{1}{\sqrt{\tau}} \sqrt{h_{k \tau}^{(n)}} \kappa_{\epsilon_{k \tau}^{\prime}}^{(n)}\left(-\sqrt{\tau} \theta_{k \tau}^{(n)}\right)\right) \tau+\kappa_{\epsilon_{k \tau}}^{(n)}\left(-\sqrt{\tau} \theta_{k \tau}^{(n)}\right) \\
& -\kappa_{\epsilon_{k \tau}}^{(n)}\left(\sqrt{\tau}\left(\sqrt{h_{k \tau}^{(n)}}-\theta_{k \tau}^{(n)}\right)\right)+\sqrt{\tau h_{k \tau}^{(n)}} \sqrt{\kappa_{\epsilon_{k \tau}}^{\prime \prime(n)}\left(-\sqrt{\tau} \theta_{k \tau}^{(n)}\right)} \epsilon_{k \tau}^{*(n)} \\
h_{k \tau}^{(n)} & =\alpha_{0}(\tau)+\alpha_{1}(\tau) h_{k \tau}^{(n)}\left(\sqrt{\kappa_{\epsilon_{k \tau}}^{\prime \prime(n)}\left(-\sqrt{\tau} \theta_{k \tau}^{(n)}\right)} \epsilon_{k \tau}^{*(n)}+\kappa_{\epsilon_{k \tau}}^{(n)}\left(-\sqrt{\tau} \theta_{k \tau}^{(n)}\right)-\gamma(\tau)\right)^{2} \\
& +\beta_{1}(\tau) h_{k \tau}^{(n)} .
\end{aligned}
$$

Here, the innovation process $\left\{\epsilon_{k \tau}^{*(n)}\right\}_{k=0, \ldots, n T}$ is a sequence of $\mathcal{F}_{(k-1) \tau}^{(n)}$-conditionally uncorrelated zero mean and unit variance $\mathbf{D}^{*}$-distributed random variables under $Q_{\text {ess }}^{(n)}$, $\epsilon_{k \tau}^{*(n)} \mid \mathcal{F}_{(k-1) \tau}^{(n)} \sim \mathbf{D}^{*}(0,1)$, related to the original innovations via the expression:

$$
\epsilon_{k \tau}^{*(n)}=\frac{\epsilon_{k \tau}^{(n)}-\kappa_{\epsilon_{k \tau}}^{(n)}\left(-\sqrt{\tau} \theta_{k \tau}^{(n)}\right)}{\sqrt{\kappa_{\epsilon_{k \tau}}^{\prime \prime(n)}\left(-\sqrt{\tau} \theta_{k \tau}^{(n)}\right)}}, \quad k=0, \ldots, n T .
$$

Here, $\kappa_{\epsilon_{k \tau}}^{\prime(n)}(\cdot)$ and $\kappa_{\epsilon_{k \tau}}^{\prime \prime(n)}(\cdot)$ are the first and second derivatives of the cgf of $\epsilon_{k \tau}$ under P. 
The innovations' law $D^{*}$ may be different than the law under $P$, although there are several cases in which the two belong to the same family. For example, when the innovations are standard Gaussian distributed, both risk neutral models of (3.2)-(3.3) and (3.8)-(3.9) coincide with the pricing model of Duan (1996) obtained via the LRNVR principle. Indeed, when $\epsilon_{k \tau}^{(n)} \sim \mathbf{N}(0,1)$ under $P^{(n)}$, it follows that $\epsilon_{k \tau}^{*(n)} \sim \mathbf{N}(0,1)$, under $Q_{\text {egp }}^{(n)}$ and $Q_{\text {ess }}^{(n)}$, and $\varrho_{k \tau}^{(n)}=\theta_{k \tau}^{(n)}=\lambda^{(\epsilon)}$. Although Duan (1996) defines the Radon-Nikodym derivative of LRNVR in terms of utility functions of consumption, the two Radon-Nikodym derivatives have the same form which corresponds to a discretized Girsanov change of measure in continuous time corresponding to a market price of risk of equity risk $\lambda^{(\epsilon)}$ and a zero price of variance risk:

$$
\left.\frac{d Q^{(n)}}{d P^{(n)}}\right|_{\mathcal{F}_{k \tau}^{(n)}}=\exp \left(\sum_{l=1}^{k}\left(-\sqrt{\tau} \lambda^{(\epsilon)} \epsilon_{k \tau}^{(n)}-\frac{1}{2} \tau\left(\lambda^{(\epsilon)}\right)^{2}\right)\right) .
$$

Indeed, in continuous time stochastic volatility models, option pricing is generally based on the Girsanov theorem (see e.g. Karatzas and Shreve (1988) or Frey (1996)). The Radon-Nykodim derivative of the family of equivalent (local) martingales measures is introduced with respect to the Brownian motion $\left\{B_{t}^{(1)}, B_{t}^{(2)}\right\}$ on $\left(\Omega, \mathcal{F},\left\{\mathcal{F}_{t}\right\}_{t \in[0, \ldots, T]}, P\right)$ as:

$$
Z_{t}(\nu):=\left.\frac{d Q}{d P}\right|_{\mathcal{F}_{t}}=\exp \left(-\sum_{i=1}^{2}\left(\int_{0}^{t} \nu_{s}^{(i)} d B_{s}^{(i)}+\frac{1}{2} \int_{0}^{t}\left(\nu_{s}^{(i)}\right)^{2} d s\right)\right) .
$$

Here, $\nu=\left\{\nu_{t}\right\}_{t \in[0, \ldots, T]}=\left\{\nu_{t}^{(1)}, \nu_{t}^{(2)}\right\}_{t \in[0, \ldots, T]}$ is a two-dimensional vector of measurable and adapted processes representing the market prices of $B_{t}^{(1)}$ and $B_{t}^{(2)}$ risks and satisfying the standard integrability constraints. The necessary and sufficient condition for the process $\left\{Z_{t}(\nu)\right\}_{t \in[0, \ldots, T]}$ to be a martingale under $P$ is that $E^{P}\left[Z_{T}(\nu)\right]=1$; this follows from various standard conditions (e.g. Novikov's condition).

In order to fully characterize the risk neutralized asset return dynamics for the associated market, we need to identify the market prices of $B_{t}^{(1)}$ and $B_{t}^{(2)}$ risks. The form of $\nu_{t}^{(1)}$ is determined from the (local) martingale constraint imposed on the discounted asset price after the change of measure, and this is illustrated in the first part of Theorem 3.3 below. The main issue remaining, however, is to find an appropriate specification for the price $\nu_{t}^{(2)}$ of non-hedgeable risk. A popular approach in the continuous time literature 
is to choose this quantity so that it satisfies some optimality criterion. For example, the minimal martingale measure is obtained by taking $\nu_{t}^{(2)}=0$ in (3.12). Another example is in Hobson (2004), which derives, for a general class of stochastic volatility models with correlated Brownian motions, an explicit form for the market price of $B_{t}^{(2)}$ risk which leads to the $q$-optimal measure.

Our aim is to characterize the class of risk neutral GARCH diffusion processes based on (3.12) and to identify the unique market prices of volatility risk, $\nu_{t}^{(2)}$, which links this model to the weak limits obtained via the extended Girsanov principle and the conditional Esscher transform. These are stated in the main result of the paper.

Proposition 3.3 Suppose that the measure $Q$ defined in (3.12) satisfies $E^{P}\left[Z_{T}(\nu)\right]=1$. Then the following statements hold:

(i) The asset return process $\left\{Y_{t}\right\}_{t \in[0, T]}$ determined by (2.4)-(2.5) is a local martingale under $Q$ and its dynamics are given by:

$$
\begin{aligned}
d Y_{t} & =\left(r-\frac{1}{2} h_{t}\right) d t+\sqrt{h_{t}} d B_{t}^{*(1)} \\
d h_{t} & =\left(\omega_{0}-\left(\omega_{1}+\sqrt{\omega_{2}}\left(M_{3}-2 \omega_{3}\right) \nu_{t}^{(1)}+\sqrt{\omega_{2}} \nu_{t}^{(2)} \sqrt{M_{4}-M_{3}^{2}-1}\right) h_{t}\right) d t \\
& +\sqrt{\omega_{2}}\left(M_{3}-2 \omega_{3}\right) h_{t} d B_{t}^{*(1)}+\sqrt{\omega_{2}} \sqrt{M_{4}-M_{3}^{2}-1} h_{t} d B_{t}^{*(2)} .
\end{aligned}
$$

Here $B_{t}^{*(1)}$ and $B_{t}^{*(2)}$ are two independent Brownian motions under $Q$ :

$$
\begin{aligned}
& B_{t}^{*(1)}=B_{t}^{(1)}+\int_{0}^{t} \nu_{s}^{(1)} d s, \\
& B_{t}^{*(2)}=B_{t}^{(2)}+\int_{0}^{t} \nu_{s}^{(2)} d s,
\end{aligned}
$$

where the market price of $B_{t}^{(1)}$ risk is given by:

$$
\nu_{t}^{(1)}=\lambda^{(\epsilon)}+\frac{\frac{1}{2} h_{t}-\kappa_{t}\left(\sqrt{h_{t}}\right)}{\sqrt{h_{t}}} .
$$

(ii) The market price of $B_{t}^{(2)}$ risk corresponding to the weak limit of the discretized risk-neutral GARCH model from (3.2)-(3.3) under $Q_{\text {egp }}^{(n)}$ is:

$$
\nu_{t}^{(2)}=-\nu_{t}^{(1)} \frac{M_{3}}{\sqrt{M_{4}-M_{3}^{2}-1}} .
$$


(iii) The market price of $B_{t}^{(2)}$ risk corresponding to the weak limit of the discretized risk-neutral GARCH model from (3.8)-(3.9) under $Q_{\text {ess }}^{(n)}$ is:

$$
\nu_{t}^{(2)}=0
$$

We notice that the market price of $B_{t}^{(1)}$ in (3.17) is obtained as the weak limit of both the mean adjustment process $\varrho^{(n)}$ of the EGP transform from $(3.5)$ and the Esscher coefficient $\theta^{(n)}$ which satisfies (3.7). In the case of the conditional Esscher transform, the risk neutralized continuous limit corresponds to that of the minimal martingale measure regardless the distribution governing the GARCH model. Since $\nu_{t}^{(2)}=0$ whenever $M_{3}=0$, the same limit is obtained in the case of the extended Girsanov principle for symmetric densities. However, in the latter case this is no longer true for skewed distributions and, according to (3.18), the non-zero market price of variance risk is proportional to the market price of equity risk where the constant of proportionality depends on the higher moments of the GARCH innovations. A negatively skewed asset return density induces therefore a positive market price of the non-hedgeable risk. Following this argument, one should expect to obtain higher SV option prices based on the EGP transform when the GARCH innovations exhibit more negative skeweness combined also with less leptokurtosis.

The above result can also be viewed as an extension of Duan's (1996) convergence theorem of locally risk neutralized Gaussian GARCH models to bivariate diffusions for a non-Gaussian framework. Indeed, when the driving noise is Gaussian, the prices of risk processes are $\nu_{t}^{(1)}=\lambda^{(\epsilon)}$ and $\nu_{t}^{(2)}=0$ for both risk neutral measures. The variance equation (3.14) reduces to the well-known GARCH diffusion process which is obtained by applying the minimal martingale measure to the process (2.4)-(2.5) based on Gaussian noise.

$$
d h_{t}=\left(\omega_{0}-\left(\omega_{1}-2 \lambda^{(\epsilon)} \sqrt{\omega_{2}} \omega_{3}\right) h_{t}\right) d t-2 \sqrt{\omega_{2}} \omega_{3} h_{t} d B_{t}^{*(1)}+\sqrt{2 \omega_{2}} h_{t} d B_{t}^{*(2)}
$$

The resulting process in (3.14) does not have a Hull-White structure since the drift is not a linear function of $h_{t}$. Instead, it has a non-linear dependence through the cumulant generating function of the GARCH noise. Using Taylor approximations for the cumulant generating function, one can obtain a different approximations for $\nu_{t}^{(1)}$. For example, we 
obtain $\nu_{t}^{(1)}=\lambda^{(\epsilon)}$ using a second order Taylor expansion for $\kappa_{\epsilon_{t}}\left(\sqrt{h_{t}}\right)$. If we include a fourth order approximation we have:

$$
\nu_{t}^{(1)}=\lambda^{(\epsilon)}-\frac{1}{6} M_{3} h_{t}-\frac{1}{24} \sqrt{h_{t}^{3}}\left(M_{4}-3\right)
$$

Equation (3.13) ensures that the discounted asset price is a local martingale under $Q$. Since the resulting limiting variance process does not come out of a Hull-White model, it is necessary to investigate carefully under what conditions $\tilde{S}_{t}$ is a true martingale. Following Sin (1995), this feature is equivalent to having a non-positive correlation between the asset return and its variance, $\operatorname{Cov}\left(d Y_{t}, d h_{t}\right) \leq 0$, provided that the market price of $B_{t}^{(1)}$ risk $\nu_{t}^{(1)}$ is bounded. Note that the latter constraint guarantees the existence of $Q$ as the integrability condition is satisfied. This can be easily verified if one uses a second order Taylor expansion for $\kappa_{\epsilon_{t}}\left(\sqrt{h_{t}}\right)$, but otherwise ones need to impose $\nu_{t}^{(1)}<\infty$. Our extensive simulation study reveals the fact that this is always the case. Finally, we have:

$$
\operatorname{Cov}\left(d Y_{t}, d h_{t}\right)=\sqrt{\omega_{2}}\left(M_{3}-2 \omega_{3}\right) \sqrt{h_{t}^{3}},
$$

which is negative whenever $M_{3}<2 \omega_{3}$. This is satisfied for financial data which generally exhibits negative skewness as $\omega_{3}>0$. Therefore, we can argue that the discounted asset price process is a true martingale under the limiting GARCH model.

\section{Numerical results}

In this section we carry out numerical experiments which support the theoretical findings concerning the convergence between option prices obtained via discrete time GARCH models and their diffusion limits. We use the NIG distribution as our candidate for the non-Gaussian driving noise. The pricing performance of threshold GARCH based option pricing models with NIG innovations (NIG-TGARCH) has been studied by Badescu et al. (2011) for European style options or by Stentoft (2008) for American options, among others.

For any $j=0, \ldots, n T$, we assume that the innovation process $\epsilon_{j \tau}^{(n)}$ is NIG distributed with parameters $k, a, s$ and $\ell$ under $P^{(n)}$, that is, $\epsilon_{j \tau}^{(n)} \sim \operatorname{NIG}(k, a, s, \ell)$, with cumulant 
generating function given by:

$$
\kappa_{\epsilon_{j \tau}}^{(n)}(z)=z \ell+\left(\sqrt{k^{2}-a^{2}}-\sqrt{k^{2}-(a+z s)^{2}}\right) .
$$

The parameter $k$ measures the degree of kurtosis of the distribution, $a$ the asymmetry, $s$ is the scale parameter, and $\ell$ is the location of the distribution. This parametrization makes the distribution a location-scale one and $k$ and $a$ are called the invariant parameters. The model parameters are obtained by fitting the GARCH model from (2.1)-(2.2) with $\tau=1$ based on the NIG distribution via maximum likelihood estimation (MLE). We use daily log-returns of the S\&P 500 index from January 4nd, 1988 to April 17th, 2002. The NIGNGARCH estimated values are: $\alpha_{0}(1)=1.7653 \cdot 10^{-6}, \alpha_{1}(1)=0.0701, \beta_{1}(1)=0.8729$, $\gamma(1)=0.8019$ and $\lambda=0.0417$. We also estimate $r=3.5433 \cdot 10^{-7}$. Additionally, the estimated values for the NIG invariant parameters are $k=1.2044$ and $a=-0.1282$. The other two parameters are found by imposing a zero mean and unit variance for the innovations, $s=1.1840$ and $l=0.1267$. These lead to the following values for the third and fourth moment of the GARCH driving noise: $M_{3}=-0.2682$ and $M_{4}=5.2118$.

In our first numerical experiment we compare the prices associated with European call options with different strikes and maturities when we assume that the dynamics of the underlying asset follows the daily NIG-GARCH process as well as its diffusion limits under the two choices of risk neutral measures. Prices are computed as discounted expected payoffs under the risk neutral measure, and since there are no closed form solutions, they are obtained via Monte-Carlo simulations based on 100,000 paths each. In the NIG-NGARCH case, this is explicitly carried out by simulating the asset return process based on equations (3.2)-(3.3) and (3.8)-(3.9) with the daily time step $\tau=1$. The limiting diffusion prices are computed according to Proposition 3.3. Sample paths are simulated using an Euler discretization of 1,024 steps per day, and the model parameters are those induced by the corresponding GARCH processes. Moreover, the last estimated conditional variance implied by the discrete-time model is used as the starting value. We notice that the discretization of the SV model never yields negative volatility values, making unnecessary the use of appropriate schemes to handle this problem (e.g. see Labbé et al. (2012) and references therein). Once both the GARCH and SV paths have been generated, they are submitted to the empirical martingale simulation (EMS) correction 
(see Duan and Simonato (1998)); this is done before we compute the expectation that provides the option price. The EMS is a high performance variance reduction method that keeps constant in time the empirical mean of the discounted prices, making the numerical path generation process consistent with the martingale condition. The results are illustrated in Tables 1 and 2 in which the call option price corresponding to each timeto-expiration and strike has been calculated 120 times. The tables report the average prices with their associated standard errors in the brackets. Although almost all prices are significantly different at $99 \%$, we notice that the absolute pricing error between GARCH and diffusions are generally smaller for shorter maturities and increase consistently for larger maturities. However, the differences in option prices are less pronounced for the Esscher transform as compared to the extended Girsanov principle. Another interesting pattern is that under both changes of measure, the discrete time model provides higher prices than its continuous time limit across all strikes and maturities. Moreover, we notice that the Esscher implied stochastic volatility limit produces higher prices than the EGP implied one, which is consistent with the general option price ordering conjecture developed in Henderson (2005) and Henderson et al. (2005) for SV models. This result states that for options with convex payoff, prices are decreasing functions in the market price of volatility risk. This is indeed verified in our case since $\nu_{t}^{(2)}=0$ in the case of Esscher and positive under EGP as $M_{3}<0$. Interestingly, the differences between the two prices are higher for ATM options and smaller for ITM and OTM options. Finally, we note that in all cases the Esscher and EGP based NIG-NGARCH call option prices are very similar contrary to the findings from Badescu et al. (2011) where option prices where computed based on a different GARCH model with the asymmetry introduced via a threshold dynamic. A more detailed discussion is found in Chorro et al. (2011b).

The second experiment that we have conducted aims at visualizing the speed of convergence of the GARCH option prices to their continuous time limit. Since the differences between EGP based daily GARCH prices and their SV limits are larger than the Esscher counterparts, we present the convergence only in the former case. We proceed with the same prescription as in the previous experiment, but this time we consider the class of risk-neutralized discrete time models from (3.2)-(3.3) that correspond to higher 


\begin{tabular}{|c|c|c|c|c|c|c|}
\hline \multicolumn{2}{|c|}{ NIG-NGARCH (daily, } & \multicolumn{5}{|c|}{ EGP) and SV Prices for European call Options $S_{0}=1000$} \\
\hline Maturity & Model & Strike $=800$ & Strike $=900$ & Strike $=1000$ & Strike $=1100$ & Strike $=1200$ \\
\hline \multirow{4}{*}{21} & \multirow{2}{*}{ GARCH-EGP } & 200.159 & 101.740 & 20.593 & 0.406 & 0.008 \\
\hline & & $(0.006)$ & $(0.008)$ & $(0.006)$ & $(0.001)$ & $(0.000)$ \\
\hline & \multirow{2}{*}{ SV-EGP } & 200.100 & 101.627 & 20.920 & 0.209 & 0.000 \\
\hline & & $(0.001)$ & $(0.003)$ & $(0.005)$ & $(0.001)$ & $(0.000)$ \\
\hline \multirow{4}{*}{63} & \multirow{2}{*}{ GARCH-EGP } & 202.671 & 109.705 & 35.972 & 4.480 & 0.316 \\
\hline & & $(0.013)$ & $(0.013)$ & $(0.010)$ & $(0.004)$ & $(0.001)$ \\
\hline & \multirow{2}{*}{ SV-EGP } & 202.550 & 109.629 & 35.895 & 4.015 & 0.152 \\
\hline & & $(0.006)$ & $(0.009)$ & $(0.009)$ & $(0.004)$ & $(0.001)$ \\
\hline \multirow{4}{*}{126} & \multirow{2}{*}{ GARCH-EGP } & 208.390 & 120.893 & 51.751 & 13.747 & 2.319 \\
\hline & & $(0.020)$ & $(0.017)$ & $(0.012)$ & $(0.007)$ & $(0.003)$ \\
\hline & \multirow{2}{*}{ SV-EGP } & 208.008 & 120.309 & 51.008 & 12.941 & 1.815 \\
\hline & & $(0.011)$ & $(0.014)$ & $(0.015)$ & $(0.009)$ & $(0.004)$ \\
\hline \multirow{4}{*}{168} & \multirow{2}{*}{ GARCH-EGP } & 212.352 & 127.574 & 60.447 & 20.345 & 4.814 \\
\hline & & $(0.020)$ & $(0.019)$ & $(0.015)$ & $(0.010)$ & $(0.005)$ \\
\hline & \multirow{2}{*}{ SV-EGP } & 211.690 & 126.617 & 59.306 & 19.244 & 4.069 \\
\hline & & $(0.013)$ & $(0.016)$ & $(0.017)$ & $(0.012)$ & $(0.006)$ \\
\hline \multirow{4}{*}{210} & \multirow{2}{*}{ GARCH-EGP } & 216.250 & 133.793 & 68.281 & 26.859 & 8.025 \\
\hline & & $(0.024)$ & $(0.021)$ & $(0.016)$ & $(0.011)$ & $(0.006)$ \\
\hline & \multirow{2}{*}{ SV-EGP } & 215.301 & 132.474 & 66.777 & 25.463 & 7.025 \\
\hline & & $(0.015)$ & $(0.019)$ & $(0.019)$ & $(0.014)$ & $(0.008)$ \\
\hline
\end{tabular}

Table 1: Comparison of the call option prices obtained using a daily NIG-NGARCH model and its diffusion limit based on the extended Girsanov principle. The prices reported have been obtained as the average of 120 Monte Carlo simulations involving 100,000 paths each with different random number generator seeds. Each Monte Carlo run has been corrected using the EMS variance reduction method. The SV has been simulated using a Euler discretization of 1,024 time steps per day. The figures in parenthesis report the standard errors of those price estimates. 


\begin{tabular}{|c|c|c|c|c|c|c|}
\hline \multicolumn{2}{|c|}{ NIG-NGARCH (daily, } & \multicolumn{5}{|c|}{ Esscher) and SV Prices for European call Options $S_{0}=1000$} \\
\hline Maturity & Model & Strike $=800$ & Strike $=900$ & Strike $=1000$ & Strike $=1100$ & Strike $=1200$ \\
\hline \multirow{4}{*}{21} & \multirow{2}{*}{ GARCH-ESS } & 200.186 & 101.766 & 20.608 & 0.406 & 0.008 \\
\hline & & $(0.007)$ & $(0.010)$ & $(0.007)$ & $(0.001)$ & $(0.000)$ \\
\hline & \multirow{2}{*}{ SV-ESS } & 200.100 & 101.647 & 20.994 & 0.215 & 0.000 \\
\hline & & $(0.001)$ & $(0.003)$ & $(0.005)$ & $(0.001)$ & $(0.000)$ \\
\hline \multirow{4}{*}{63} & \multirow{2}{*}{ GARCH-ESS } & 202.740 & 109.758 & 35.993 & 4.482 & 0.316 \\
\hline & & $(0.012)$ & $(0.013)$ & $(0.009)$ & $(0.004)$ & $(0.001)$ \\
\hline & \multirow{2}{*}{ SV-ESS } & 202.650 & 109.861 & 36.229 & 4.159 & 0.166 \\
\hline & & $(0.006)$ & $(0.009)$ & $(0.010)$ & $(0.005)$ & $(0.001)$ \\
\hline \multirow{4}{*}{126} & \multirow{2}{*}{ GARCH-ESS } & 208.506 & 120.990 & 51.805 & 13.770 & 2.323 \\
\hline & & $(0.016)$ & $(0.015)$ & $(0.012)$ & $(0.007)$ & $(0.004)$ \\
\hline & \multirow{2}{*}{ SV-ESS } & 208.419 & 120.948 & 51.735 & 13.411 & 1.957 \\
\hline & & $(0.012)$ & $(0.014)$ & $(0.015)$ & $(0.010)$ & $(0.004)$ \\
\hline \multirow{4}{*}{168} & \multirow{2}{*}{ GARCH-ESS } & 212.483 & 127.699 & 60.537 & 20.381 & 4.824 \\
\hline & & $(0.019)$ & $(0.016)$ & $(0.014)$ & $(0.010)$ & $(0.005)$ \\
\hline & \multirow{2}{*}{ SV-ESS } & 212.304 & 127.491 & 60.270 & 19.948 & 4.369 \\
\hline & & $(0.014)$ & $(0.016)$ & $(0.017)$ & $(0.013)$ & $(0.006)$ \\
\hline \multirow{4}{*}{210} & \multirow{2}{*}{ GARCH-ESS } & 216.431 & 133.973 & 68.417 & 26.927 & 8.049 \\
\hline & & $(0.020)$ & $(0.017)$ & $(0.016)$ & $(0.012)$ & $(0.007)$ \\
\hline & \multirow{2}{*}{ SV-ESS } & 216.135 & 133.583 & 67.971 & 26.378 & 7.505 \\
\hline & & $(0.016)$ & $(0.019)$ & $(0.018)$ & $(0.014)$ & $(0.008)$ \\
\hline
\end{tabular}

Table 2: Comparison of the call option prices obtained using a daily NIG-NGARCH model and its diffusion limit based on the conditional Esscher transform. The prices reported have been obtained as the average of 120 Monte Carlo simulations involving 100,000 paths each with different random number generator seeds. Each Monte Carlo run has been corrected using the EMS variance reduction method. The SV has been simulated using a Euler discretization of 1,024 time steps per day. The figures in parenthesis report the standard errors of those price estimates. 
and higher sampling frequencies. In other words, we compute European call option prices for different values of $\tau$, where $\tau=1, \frac{1}{2}, \frac{1}{2^{2}}, \ldots$ The GARCH parameters are adjusted accordingly from the daily estimated values and the starting variance is the same as in the previous study. The continuous time "true" price limit is computed using the Euler scheme based on 1,024 steps per day. In Figure 1 we represent graphically the square of the difference between the NIG-NGARCH and the SV based prices based on the EGP transform as a function of the strike and the logarithm of the number of intraday time steps considered; we call this amount the mean square pricing errors (MSPE). This is performed for maturities of 21, 63, 126 and 168 days. We notice that the pictures are generally smooth (especially for shorter-dated options) and the MSPEs converge to zero as the GARCH sampling frequency goes to infinity. In fact, the convergence is fast and we only need a reasonably small number of intra daily steps to obtain an MSPE very close to zero. However, the number of steps increases with the time-to-expiration of the options considered. The numerical convergence becomes slightly less smooth in the case of longer-dated options such as $T=168$ days. An important point to emphasize here is the role of using the Empirical Martingale Simulation (EMS) variance reduction technique of Duan in this exercise, because without it, and using a tractable number of paths in the simulation, the Monte Carlo error is approximately of the same order as the price differences that we set out to study. This is indeed crucial for observing the convergence of long dated contracts.

Finally, we perform a small calibration exercise in order to test the performance of the NIG-NGARCH models and their SV limits relative to the observed market quotes. The same option data set used in Badescu et al. (2011) which consists of 54 European call option prices written on S\&P 500 with 5 maturities, $T=22,46,109,173$ and 234 . We divide the data set into three time-to-expiration categories: ITM options $\left(K / S_{0} \leq 0.98\right)$, $\operatorname{ATM}\left(0.98<K / S_{0}<1.02\right)$ and $\operatorname{OTM}\left(K / S_{0} \geq 1.02\right)$, so we end up with an equal number of contracts for each group. In each scenario, we calibrate the model parameters by minimizing the Average Relative Pricing Error (ARPE), defined as the absolute difference between market and model prices divided by market price, under both Esscher and EGP. The ARPEs are illustrated in Table 3 for each class of moneyness. We 



Figure 1: Convergence of the EGP based NIG-NGARCH option prices to their continuous time limit counterparts for different maturities as a function of the strike $\left(S_{0}=1000\right)$ and the number of intraday time steps considered when setting up the GARCH model frequency.

also report the overall pricing errors which are computed from calibrating our models to all contracts. We notice that for both continuous and discrete time models the EGP

\begin{tabular}{lcccc}
\hline \multicolumn{4}{c}{ Average Relative Pricing Errors (ARPE) } \\
\cline { 2 - 4 } Model & {$[0.86,0.98]$} & {$[0.98,1.02]$} & {$[1.02,1.20]$} & Overall \\
\cline { 2 - 5 } GARCH-EGP & $0.54 \%$ & $0.74 \%$ & $1.19 \%$ & $1.41 \%$ \\
GARCH-ESS & $0.51 \%$ & $0.89 \%$ & $1.64 \%$ & $1.42 \%$ \\
SV-EGP & $0.46 \%$ & $0.31 \%$ & $1.31 \%$ & $1.29 \%$ \\
SV-ESS & $0.63 \%$ & $0.64 \%$ & $1.61 \%$ & $1.32 \%$ \\
\hline
\end{tabular}

Table 3: Average relative pricing errors (ARPE) from the calibration of the NIG-NGARCH models and their diffusion limits to European call options on S\&P 500 for different groups of moneyness. The overall ARPE is computed from calibrating all contracts. 
pricing kernel slightly outperforms its Esscher counterpart, the largest differences being observed for out-of-money options. The presence of a non-zero market price of volatility risk (the SV-EGP case) reduces the ARPE for all groups of moneyness in the case of diffusions. Finally, in Table 4 we report the skewness of the GARCH innovations implied by the observed option quotes. We notice that the skewness obtained from calibrating

Skewness of NIG-NGARCH innovations

\begin{tabular}{lcccc}
\hline \multirow{4}{*}{ Model } & \multicolumn{3}{c}{ Moneyness $S_{0} / K$} & \\
\cline { 2 - 4 } & {$[0.86,0.98]$} & {$[0.98,1.02]$} & {$[1.02,1.20]$} & Overall \\
\hline GARCH-EGP & 0.04 & -0.08 & -0.11 & -0.13 \\
GARCH-ESS & -0.16 & -0.13 & -0.13 & -0.13 \\
SV-EGP & -0.25 & -0.25 & -0.25 & -0.25 \\
SV-ESS & -0.25 & -0.25 & -0.25 & -0.25 \\
\hline
\end{tabular}

Table 4: Skewness of the NIG-NGARCH innovations implied by European call options on S\&P 500 for different groups of moneyness. The overall skewness is computed from calibrating all contracts.

the SV models is constant for all groups and is equal to -0.25 . This is due to the fact that the implied parameters of the NIG distribution are quite stable and do not change within each moneyness class considered. Therefore, only the changes in the calibrated GARCH parameters affect the pricing performance of these models. This is no longer the case for the NIG-NGARCH option pricing models, where the NIG distribution parameters slightly change across maturities, especially for the EGP pricing kernel. Since our calibration exercise is performed on options observed on only one day, we believe that the effect of the skenewss and leptokurtosis of the underlying distribution on the pricing model/methodology needs to be further tested on larger option data sets.

\section{Conclusions}

In this paper we theoretically investigate the continuous time limits of a general nonGaussian GARCH model with application to pricing European style options. Since 
Duan's (1995) LRNVR is no longer applicable in this setting we propose the use of the extended Girsanov principle and the conditional Esscher transform as our pricing kernel candidates. Applying this changes of measures to a discretized asymetric GARCH model we obtained two risk neutral processes which converge to two different stochastic volatility models. Both continuous time limits can be obtained by applying the Girsanov theorem to the GARCH diffusion under the physical measure for various choices of market prices of variance risk. For example, for any GARCH innovation distribution, the Esscher implied SV model is obtained from the Girsanov change of measure based on a zero market price of volatility risk, and this corresponds to the minimal martingale measure. However, when asset returns exhibit skewness, the extended Girsanov implied GARCH diffusion is obtained with a non-zero market price of variance risk which is proportional to the market price of equity risk, the constant of proportionality depending on the third and the fourth moment of the underlying distribution. The two time limits coincide in the case of Gaussian driven GARCH models or, moreover, for any process governed by a symmetric density. Our theoretical results are further supported by several simulation exercises. When comparing daily NIG-GARCH and their continuous limits for European call option prices we observe that there are small differences across all maturities and moneyness considered. The GARCH option prices based on the Esscher transform are closer to their SV counterparts than those based on the EGP pricing kernel. We also notice that for the SV limits, the presence of a non-zero market price of variance risk in the case of EGP gives rise to lower option prices than the Esscher implied ones, which is consistent with the theoretical price ordering results previously developed in the literature. The convergence is also illustrated for the extended Girsanov principle by computing option prices for difference sampling frequencies. Finally, a small size calibration exercise on observed option quotes on the S\&P 500 index indicates that the presence of a variance price of risk slightly improves the overall pricing performance of the GARCH models and their diffusion limits. We further observe that the parameters of the innovation distribution implied by the option prices remain stable across all groups of moneyness considered, which is not the case for the NIG-NGARCH option pricing models based on the EGP. 


\section{References}

Alexander, C. and Lazar, E. (2005). On the continuous limit of GARCH. ICMA Centre Discussion Papers in Finance 13.

Badescu, A., Elliott, R. J., Kulperger, R., Jarkko, M. and Siu, T. K. (2011). A comparison of pricing kernels for GARCH option pricing with generalized hyperbolic distributions. International Journal of Theoretical and Applied Finance 14, 669-708.

Badescu, A. M. and Kulperger, R. J. (2008). GARCH option pricing: A semiparametric approach. Insurance: Mathematics and Economics 43, 69-84.

Badescu, A. M., Kulperger, R. J. and Lazar, E. (2008). Option valuation with Normal Mixture GARCH models. Studies in Nonlinear Dynamics and Econometrics 12, Article 5.

Bollerslev, T. (1986). Generalized autoregressive conditional heteroskedasticity. Journal of Econometrics 31, 307-327.

Chorro, C., Guegan, D. and Ielpo, F. (2011a). Option pricing for GARCH-type models with generalized hyperbolic innovations. Quantitative Finance 12, 1079-1094.

Chorro, C., Guegan, D. and Ielpo, F. (2011b). Martingalized historical approach for option pricing. Finance Research Letters 7, 24-28.

Christoffersen, P., Dorion, C., Jacobs, K. and Wang, Y. (2010). Volatility components, affine restrictions, and nonnormal innovations. Journal of Business \& Economic Statistics 28, 483-502.

Christoffersen, P., Elkamhi, R., Feunou, B. and Jacobs, K. (2009). Option valuation with conditional heteroskedasticity and nonnormality. Review of Financial Studies 23, 2139-2183.

Christoffersen, P., Heston, S. L. and Jacobs, K. (2013a). Capturing option anomalies with a variance-dependent pricing kernel. Review of Financial Studies In press. 
Christoffersen, P., Jacobs, K. and Ornthanalai, C. (2013b). GARCH option valuation: theory and evidence. Working paper.

Corradi, V. (2000). Reconsidering the continuous time limit of the $\operatorname{GARCH}(1,1)$ process. Journal of Econometrics 96, 145-153.

Duan, J.-C. (1995). The GARCH option pricing model. Mathematical Finance 5, 13-32.

Duan, J.-C. (1996). A unified theory of option pricing under stochastic volatility: from GARCH to diffusion. Working paper.

Duan, J.-C. (1997). Augmented GARCH(p,q) process and its diffusion limit. Journal of Econometrics 79, 97-127.

Duan, J.-C., Ritchken, P. and Sun, Z. (2006). Approximating GARCH-jump models, jump-diffusion processes, and option pricing. Mathematical Finance 16, 21-52.

Duan, J.-C. and Simonato, J.-G. (1998). Empirical martingale simulation of asset prices. Management Science 44, 1218-1233.

Duan, J.-C., Wang, Y. and Zou, J. (2009). Convergence speed of GARCH option price to diffusion option price. International Journal of Theoretical and Applied Finance 12, $359-391$.

Elliott, R. J. and Madan, D. B. (1998). A discrete time equivalent martingale measure. Mathematical Finance 8, 127-152.

Engle, R. F. (1982). Autoregressive conditional heteroscedasticity with estimates of the variance of United Kingdom inflation. Econometrica 50, 987-1007.

Fornari, F. and Mele, A. (1997). Weak convergence and distributional assumptions for a general class of nonlinear ARCH models. Econometric Reviews 16, 205-227.

Frey, R. (1996). Derivative asset analysis in models with level-dependent and stochastic volatility. CWI QUARTERLY 10, 1-34.

Henderson, V. (2005). Analytical comparisons of options prices in stochastic volatility models. Mathematical Finance 15, 49-59. 
Henderson, V., Hobson, D., Howison, S. and Kluge, T. (2005). Analytical comparisons of options prices in stochastic volatility models. Review of Derivatives Research 8, 5-25.

Heston, S. L. and Nandi, S. (2000). A closed-form GARCH option valuation model. The Review of Financial Studies 13, 585-625.

Hobson, D. (2004). Stochastic volatility models, correlation and the q-optimal measure. Mathematical Finance 14, 537-556.

Hull, J. and White, A. (1987). The pricing of options on assets with stochastic volatilities. The Journal of Finance 42, 281-300.

Karatzas, I. and Shreve, S. E. (1988). Brownian Motion and Stochastic Calculus, vol. 113, of Graduate Texts in Mathematics. Springer US, New York, NY.

Labbé, C., Rémillard, B. and Renaud, J.-F. (2012). A simple discretization scheme for nonnegative diffusion processes with application to option pricing. The Journal of Computational Finance 15, 3-35.

Lindner, A. (2009). Continuous time approximations to GARCH and stochastic volatility models. In Handbook of Financial Time Series, (Andersen, T. G., Davis, R. A., Kreiß, J.-P. and Mikosch, T., eds),. Springer-Verlag.

Menn, C. and Rachev, S. (2005). A GARCH option pricing model with a-stable innovations. European Journal of Operational Research 163, 201-209.

Monfort, A. and Pegoraro, F. (2012). Asset pricing with Second-Order Esscher Transforms. Journal of Banking \& Finance 36, 1678-1687.

Nelson, D. B. (1990). ARCH models as diffusion approximations. Journal of Econometrics $45,7-38$.

Prigent, J. L. and Scaillet, O. (2002). Weak convergence of hedging strategies of contingent claims.

Siu, T. K., Tong, H. and Yang, H. (2004). On pricing derivatives under GARCH models: a dynamic Gerber-Shiu approach. North American Actuarial Journal 8, 17-32. 
Stentoft, L. (2008). American option pricing using GARCH models and the Normal Inverse Gaussian distribution. Journal of Financial Econometrics 6, 540-582.

Stentoft, L. (2011). American option pricing with discrete and continuous time models: An empirical comparison. Journal of Empirical Finance 18, 880-902.

Stroock, D. W. and Varadhan, S. R. S. (1979). Multidimensional diffusion processes. Springer-Verlag.

\section{Appendix}

\subsection{Proof of Proposition 2.1}

The proof follows from the convergence of Markov processes to diffusions, (see e.g. Stroock and Varadhan (1979). We calculate the limits as $\tau$ approaches zero of the first conditional moments and the covariance matrix of the underlying discretized GARCH process from (2.1)-(2.2) needed for identifying the coefficients of the continuous time bivariate model.

Thus, for any $k=0, \ldots, n T$, and using the parametric constraints from Proposition 2.1, the drift coefficients are obtained from:

$$
\begin{aligned}
\lim _{\tau \rightarrow 0} \frac{1}{\tau} \mathrm{E}^{P^{(n)}}\left[\Delta Y_{k \tau}^{(n)} \mid \mathcal{F}_{(k-1) \tau}^{(n), h_{t}}\right] & =r+\lambda^{(\epsilon)} \sqrt{h_{t}}-\kappa_{\epsilon_{t}}^{(n)}\left(\sqrt{h_{t}}\right), \\
\lim _{\tau \rightarrow 0} \frac{1}{\tau} \mathrm{E}^{P^{(n)}}\left[\Delta h_{(k+1) \tau}^{(n)} \mid \mathcal{F}_{(k-1) \tau}^{(n), h_{t}}\right] & =\omega_{0}-\omega_{1} h_{t} .
\end{aligned}
$$

The limiting conditional second moments of the GARCH process are obtained below:

$$
\begin{aligned}
\lim _{\tau \rightarrow 0} \frac{1}{\tau} \mathrm{E}^{P^{(n)}}\left[\left(\Delta Y_{k \tau}^{(n)}\right)^{2} \mid \mathcal{F}_{(k-1) \tau}^{(n), h_{t}}\right] & =h_{t}, \\
\lim _{\tau \rightarrow 0} \frac{1}{\tau} \mathrm{E}^{P^{(n)}}\left[\left(\Delta h_{(k+1) \tau}^{(n)}\right)^{2} \mid \mathcal{F}_{(k-1) \tau}^{(n), h_{t}}\right] & =\omega_{2}\left(M_{4}-1+4 \omega_{3}^{2}-4 M_{3} \omega_{3}\right) h_{t}, \\
\lim _{\tau \rightarrow 0} \frac{1}{\tau} \mathrm{E}^{P^{(n)}}\left[\Delta Y_{k \tau}^{(n)} \Delta h_{(k+1) \tau}^{(n)} \mid \mathcal{F}_{(k-1) \tau}^{(n), h_{t}}\right] & =\sqrt{\omega_{2}}\left(M_{3}-2 \omega_{3}\right) \sqrt{h_{t}^{3}} .
\end{aligned}
$$

The diffusion coefficients from (2.5) are obtained based on the Cholesky decomposition of the covariance matrix formed with the above elements: 


\subsection{Proof of Proposition 3.1}

Rewriting the multiplicative Doob decomposition in an additive form, we have:

$$
Y_{k \tau}^{(n)}-Y_{(k-1) \tau}^{(n)}=r \tau+\eta_{k \tau}^{(n)}+\log W_{k \tau}^{(n)}, \quad k=0, \ldots, n T .
$$

After some algebraic manipulation, we can now express the Radon-Nikodym derivative of the extended Girsanov principle from (3.1) in terms of the p.d.f. of the GARCH driving noise:

$$
Z_{k \tau}^{(n)}=\prod_{l=1}^{k} \frac{g_{W_{l \tau}}^{(n)}\left(\frac{\widetilde{S}_{l \tau}^{(n)}}{\widetilde{S}_{(l-1) \tau}^{(n)}}\right) e^{\eta_{l \tau}^{(n)}}}{g_{W_{l \tau}}^{(n)}\left(e^{-\eta_{l \tau}^{(n)}} \frac{\widetilde{S}_{l \tau}^{(n)}}{\widetilde{S}_{(l-1) \tau}^{(n)}}\right)}=\prod_{l=1}^{k} \frac{f_{\epsilon_{l \tau}}^{(n)}\left(\epsilon_{k \tau}^{(n)}+\sqrt{\tau} \varrho_{k \tau}^{(n)}\right)}{f_{\epsilon_{l \tau}}^{(n)}\left(\epsilon_{k \tau}^{(n)}\right)}
$$

Here, $\left\{\varrho_{k \tau}^{(n)}\right\}_{k=0, \ldots, n T}$ is the $\mathcal{F}_{k \tau}^{(n)}$ - predictable process defined in (3.5). We next show that $\left\{\epsilon_{k \tau}^{(n)}\right\}_{k=0, \ldots, n T}$ under $P^{(n)}$ and $\left\{\epsilon_{k \tau}^{*(n)}\right\}_{k=0, \ldots, n T}$, defined by $\epsilon_{k \tau}^{*(n)}=\epsilon_{k \tau}^{(n)}+\sqrt{\tau} \varrho_{k \tau}^{(n)}$, under $Q_{e g p}^{(n)}$ are conditionally identically distributed by computing the corresponding moment generating functions assuming they exist. For any $z \in(-u, u)$ using Bayes' rule, we have:

$$
\begin{aligned}
\exp \left(\kappa_{\epsilon_{k \tau}^{*}}^{*(n)}(z)\right) & =\mathrm{E}^{Q_{e g p}^{(n)}}\left[\exp \left(z \epsilon_{k \tau}^{*(n)}\right) \mid \mathcal{F}_{(k-1) \tau}^{(n)}\right]=\mathrm{E}^{Q_{e g p}^{(n)}}\left[\exp \left(z\left(\epsilon_{k \tau}^{(n)}+\sqrt{\tau} \varrho_{k \tau}^{(n)}\right)\right) \mid \mathcal{F}_{(k-1) \tau}^{(n)}\right] \\
& =\int \exp \left(z\left(x+\sqrt{\tau} \varrho_{k \tau}^{(n)}\right)\right) \frac{f_{\epsilon_{k \tau}}^{(n)}\left(x+\sqrt{\tau} \varrho_{k \tau}^{(n)}\right)}{f_{\epsilon_{k \tau}}^{(n)}(x)} f_{\epsilon_{k \tau}}^{(n)}(x) d x=\exp \left(\kappa_{\epsilon_{k \tau}}^{(n)}(z)\right) .
\end{aligned}
$$

Here, $\kappa_{\epsilon_{k \tau}^{*}}^{*(n)}(\cdot)$ is the conditional cumulant generating function of $\epsilon_{k \tau}^{*(n)}$ under $Q_{e g p}^{(n)}$. In particular, by taking the first and second order derivatives of the cumulant generating functions at zero, we notice that $\mathrm{E}^{Q_{e g p}^{(n)}}\left[\epsilon_{k \tau}^{*(n)} \mid \mathcal{F}_{(k-1) \tau}^{(n)}\right]=0$ and $\operatorname{Var}^{Q_{e g p}^{(n)}}\left[\epsilon_{k \tau}^{*(n)} \mid \mathcal{F}_{(k-1) \tau}^{(n)}\right]=1$. To show that $\epsilon_{k \tau}^{*(n)}$ are uncorrelated under $Q_{e g p}^{(n)}$ suppose $1<s<l<k \leq n T$. We have:

$$
\mathrm{E}^{Q_{e g p}^{(n)}}\left[\epsilon_{k \tau}^{*(n)} \epsilon_{(k-s) \tau}^{*(n)} \mid \mathcal{F}_{(k-l) \tau}^{(n)}\right]=E^{Q_{e g p}^{(n)}}\left[E^{Q_{e g p}^{(n)}}\left[\epsilon_{k \tau}^{*(n)} \epsilon_{(k-s) \tau}^{*(n)} \mid \mathcal{F}_{(k-1) \tau}^{(n)}\right] \mid \mathcal{F}_{(k-l) \tau}^{(n)}\right]=0 .
$$

Thus, the risk neutral innovation process is obtained by shifting the underlying driving noise by $\varrho_{k \tau}^{(n)}$. From equations (2.1)-(2.2) together with (3.5) we have the following $Q_{e g p}^{(n)}$ dynamics:

$$
\begin{aligned}
Y_{k \tau}^{(n)}-Y_{(k-1) \tau}^{(n)} & =\left(r+\lambda^{(\epsilon)} \sqrt{h_{k \tau}^{(n)}}-\kappa_{\epsilon_{k \tau}}^{(n)}\left(\sqrt{h_{k \tau}^{(n)}}\right)\right) \tau+\sqrt{\tau h_{k \tau}^{(n)}} \epsilon_{k \tau}^{(n)} \\
& =\left(r+\lambda^{(\epsilon)} \sqrt{h_{k \tau}^{(n)}}-\kappa_{\epsilon_{k \tau}}^{(n)}\left(\sqrt{h_{k \tau}^{(n)}}\right)\right) \tau \sqrt{\tau h_{k \tau}^{(n)}}\left(\epsilon_{k \tau}^{*(n)}-\sqrt{\tau} \varrho_{k \tau}^{(n)}\right) \\
& =\left(r-\frac{1}{\tau} \kappa_{\epsilon_{k \tau}}^{(n)}\left(\sqrt{\tau h_{k \tau}^{(n)}}\right)\right) \tau+\sqrt{\tau h_{k \tau}^{(n)}} \epsilon_{k \tau}^{*(n)}
\end{aligned}
$$


The risk neutral property follows by verifying that the discounted asset prices satisfy the martingale property under $Q_{e g p}^{(n)}$. Indeed,

$$
\begin{aligned}
\mathrm{E}^{Q_{e g p}^{(n)}}\left[\exp \left(\Delta Y_{k \tau}^{(n)}\right) \mid \mathcal{F}_{(k-1) \tau}^{(n)}\right] & =\mathrm{E}^{Q_{e g p}^{(n)}}\left[\left(\left(r-\frac{1}{\tau} \kappa_{\epsilon_{k \tau}}^{(n)}\left(\sqrt{\tau h_{k \tau}^{(n)}}\right)\right) \tau+\sqrt{\tau h_{k \tau}^{(n)}} \epsilon_{k \tau}^{*(n)}\right) \mid \mathcal{F}_{(k-1) \tau}^{(n)}\right] \\
& =\exp (r \tau) .
\end{aligned}
$$

Replacing $\epsilon_{k \tau}^{(n)}$ by $\epsilon_{k \tau}^{*(n)}-\sqrt{\tau} \varrho_{k \tau}^{(n)}$ into the conditional variance equation (2.2) concludes the proof.

\subsection{Proof of Proposition 3.2}

First, we compute the one-step conditional mean of $Z_{k \tau}^{(n)}$ under $P^{(n)}$ :

$$
\mathrm{E}^{P^{(n)}}\left[Z_{k \tau}^{(n)} \mid \mathcal{F}_{(k-1) \tau}^{(n)}\right]=\mathrm{E}^{P^{(n)}}\left[\exp \left(-\sqrt{\tau} \theta_{k \tau}^{(n)} \epsilon_{k \tau}^{(n)}-\kappa_{\epsilon_{k \tau}}^{(n)}\left(-\sqrt{\tau} \theta_{k \tau}^{(n)}\right)\right) \mid \mathcal{F}_{(k-1) \tau}^{(n)}\right]=1 .
$$

The martingale property of $Z^{(n)}$ follows by verifying that for any $k=0, \ldots, n T$ we have:

$$
\mathrm{E}^{P^{(n)}}\left[Z_{k \tau}^{(n)} \mid \mathcal{F}_{(k-1) \tau}^{(n)}\right]=Z_{(k-1) \tau}^{(n)} \mathrm{E}^{P^{(n)}}\left[Z_{k \tau}^{(n)} \mid \mathcal{F}_{(k-1) \tau}^{(n)}\right]=Z_{(k-1) \tau}^{(n)} .
$$

The fact that $Q_{\text {ess }}^{(n)}$ is an equivalent probability measure with respect to $P^{(n)}$ results from $\mathrm{E}^{Q_{e s s}^{(n)}}[1]=\mathrm{E}^{P^{(n)}}\left[Z_{n T}^{(n)}\right]=\mathrm{E}^{P^{(n)}}\left[Z_{0}^{(n)}\right]=1$. Finally, we show (3.7) is a sufficient condition for the discounted asset price to be a martingale under $Q_{e s s}^{(n)}$. This is equivalent to:

$$
\begin{aligned}
\mathrm{E}^{\mathrm{Q}_{\text {ess }}^{(\mathrm{n})}}\left[\exp \left(\Delta Y_{k \tau}^{(n)}\right) \mid \mathcal{F}_{(k-1) h, n}^{(n)}\right] & =\mathrm{E}^{P^{(n)}}\left[\exp \left(\Delta Y_{k \tau}^{(n)}\right) Z_{k \tau}^{(n)} \mid \mathcal{F}_{(k-1) \tau}^{(n)}\right] \\
& =\exp \left(\left(r+\lambda^{(\epsilon)} \sqrt{h_{k \tau}^{(n)}}-\kappa_{\epsilon_{k \tau}}^{(n)}\left(\sqrt{h_{k \tau}^{(n)}}\right)\right) \tau-\kappa_{\epsilon_{k \tau}}^{(n)}\left(-\sqrt{\tau} \theta_{k \tau}^{(n)}\right)\right) \\
& \times \mathrm{E}^{P^{(n)}}\left[\exp \left(\sqrt{\tau}\left(\sqrt{h_{k \tau}^{(n)}}-\theta_{k \tau}^{(n)}\right) \epsilon_{k \tau}^{(n)}\right) \mid \mathcal{F}_{(k-1) h, n}^{(n)}\right]=\exp (r \tau) .
\end{aligned}
$$

Taking the natural logarithm of the last equality in the above expression we obtain (3.7).

We now compute the conditional cumulant generating function of $\epsilon_{k \tau}^{(n)}$ under $Q_{e s s}^{(n)}$ :

$$
\begin{aligned}
\kappa_{\epsilon_{k \tau}}^{*(n)}(u) & =\log \mathrm{E}^{Q_{e s s}^{(n)}}\left[\exp \left(u \epsilon_{k \tau}^{(n)}\right) \mid \mathcal{F}_{(k-1) \tau}^{(n)}\right] \\
& =\log \mathrm{E}^{P^{(n)}}\left[\exp \left(u \epsilon_{k \tau}^{(n)}-\sqrt{\tau} \theta_{k \tau}^{(n)} \epsilon_{k \tau}^{(n)}-\kappa_{\epsilon_{k \tau}}^{(n)}\left(-\sqrt{\tau} \theta_{k \tau}^{(n)}\right)\right) \mid \mathcal{F}_{t-1}\right] \\
& =\kappa_{\epsilon_{k \tau}}^{(n)}\left(u-\sqrt{\tau} \theta_{k \tau}^{(n)}\right)-\kappa_{\epsilon_{k \tau}}^{(n)}\left(-\sqrt{\tau} \theta_{k \tau}^{(n)}\right) .
\end{aligned}
$$


Using the above and the relations between the first two central moments and cumulants we have:

$$
\begin{aligned}
\mathrm{E}^{Q_{e s s}^{(n)}}\left[\epsilon_{k \tau}^{(n)} \mid \mathcal{F}_{(k-1) \tau}^{(n)}\right] & =\kappa_{\epsilon_{k \tau}}^{*(n)}(0)=\kappa_{\epsilon_{k \tau}}^{(n)}\left(-\sqrt{\tau} \theta_{k \tau}^{(n)}\right), \\
\operatorname{Var}^{Q_{e s s}^{(n)}}\left[\epsilon_{k \tau}^{(n)} \mid \mathcal{F}_{(k-1) \tau}^{(n)}\right] & =\kappa_{\epsilon_{k \tau}}^{* \prime \prime(n)}(0)=\kappa_{\epsilon_{k \tau}}^{\prime \prime(n)}\left(-\sqrt{\tau} \theta_{k \tau}^{(n)}\right) .
\end{aligned}
$$

Thus, we obtain that $\mathrm{E}^{Q_{e s s}^{(n)}}\left[\epsilon_{k \tau}^{*(n)} \mid \mathcal{F}_{(k-1) \tau}^{(n)}\right]=0$ and $\operatorname{Var}^{Q_{e s s}^{(n)}}\left[\epsilon_{k \tau}^{*(n)} \mid \mathcal{F}_{(k-1) \tau}^{(n)}\right]=1$. Using the double expectation rule we obtain that the innovations are conditionally uncorrelated:

$\mathrm{E}^{Q_{e s s}^{(n)}}\left[\epsilon_{k \tau}^{*(n)} \epsilon_{(k-s) \tau}^{*(n)} \mid \mathcal{F}_{(k-l) \tau}^{(n)}\right]=\mathrm{E}^{Q_{e s s}^{(n)}}\left[\epsilon_{(k-s) \tau}^{*(n)} \mathrm{E}^{Q_{e s s}^{(n)}}\left[\epsilon_{k \tau}^{*(n)} \mid \mathcal{F}_{(k-1) \tau}^{(n)}\right] \mid \mathcal{F}_{(k-l) \tau}^{(n)}\right]=0, \quad 1<s<l<k$.

Finally, the risk neutral dynamics from (3.8)-(3.9) are obtained by expressing $\epsilon_{k \tau}^{(n)}$ in terms of $\epsilon_{k \tau}^{*(n)}$ into both (2.1) and (2.2) and by applying the martingale constraint (3.7).

\subsection{Proof of Proposition 3.3}

(i) The local martingale property and the risk neutral dynamics of the limiting asset return process from (3.13)-(3.14) follow from Girsanov's theorem.

(ii) Our aim is to identify the two prices of risk processes $\nu_{t}^{(1)}$ and $\nu_{t}^{(2)}$ which then links this model to the discretized discretized GARCH model under $Q^{(n)}$ from (3.2)-(3.3) via weak convergence. Thus, we need to compute the limits when $\tau$ approaches zero of the same quantities as in Proposition 2.1, but this time under the martingale measure given by the extended Girsanov principle.

First, notice that the mean adjustment process $\left\{\varrho_{k \tau}^{(n)}\right\}_{k=0, \ldots, n T}$ defined in (3.5) converges weakly to $\left\{\nu_{t}^{(1)}\right\}_{t \in[0, \ldots, T]}$ from (3.17). Indeed, since $h_{k \tau}^{(n)}$ converges to $h_{t}$ and the cumulant generating function is bounded, it follows that $\kappa_{\epsilon_{k \tau}}^{(n)}\left(\sqrt{h_{k \tau}^{(n)}}\right)$ converges to $\kappa_{\epsilon_{t}}\left(\sqrt{h_{t}}\right)$. Now, using the Taylor expansion for $\kappa_{\epsilon_{k \tau}}^{(n)}\left(\sqrt{\tau h_{k \tau}^{(n)}}\right)$ around the origin we have:

$$
\kappa_{\epsilon_{k \tau}}^{(n)}\left(\sqrt{\tau h_{k \tau}^{(n)}}\right)=\frac{1}{2} \tau h_{k \tau}^{(n)}+o(\tau), \quad k=0, \ldots, n T .
$$

Therefore, we can conclude that:

$$
\varrho_{t}:=\lim _{\tau \rightarrow 0} \varrho_{k \tau}^{(n)}=\nu_{t}^{(1)}=\lambda^{(\epsilon)}+\frac{\frac{1}{2} h_{t}-\kappa_{t}\left(\sqrt{h_{t}}\right)}{\sqrt{h_{t}}} .
$$


Note that this limit and the ones following in (iii) are considered given the filtration $\mathcal{F}_{t}^{(n), h_{t}}$. We now compute the first conditional moments under $Q^{(n)}$ needed for the drift coefficients:

$$
\begin{aligned}
\lim _{\tau \rightarrow 0} \frac{1}{\tau} \mathrm{E}^{Q_{e g p}^{(n)}}\left[\Delta Y_{k \tau}^{(n)} \mid \mathcal{F}_{(k-1) \tau}^{(n), h_{t}}\right] & =r-\frac{1}{2} h_{t}, \\
\lim _{\tau \rightarrow 0} \frac{1}{\tau} \mathrm{E}^{Q_{e g p}^{(n)}}\left[\Delta h_{(k+1) \tau}^{(n)} \mid \mathcal{F}_{(k-1) \tau}^{(n), h_{t}}\right] & =\omega_{0}-\left(\omega_{1}-2 \sqrt{\omega_{2}} \omega_{3} \nu_{t}^{(1)}\right) h_{t} .
\end{aligned}
$$

Notice that replacing (3.18) into (3.14) leads to the same risk neutral drift of the variance process. Finally, after some algebraic manipulations, the expressions for the second conditional moments lead to the same values as under $P^{(n)}$. This completes the proof for the EGP case.

(iii) We follow the same methodology as in (ii). First, we calculate some useful limits using the following Taylor expansions around the origin for the cumulant generating function of the innovation and its first two derivatives:

$$
\begin{array}{r}
\kappa_{\epsilon_{k \tau}}^{(n)}(u)=\kappa_{\epsilon_{k \tau}}^{(n)}(0)+u \kappa_{\epsilon_{k \tau}}^{\prime(n)}(0)+\frac{u^{2}}{2} \kappa_{\epsilon_{k \tau}}^{\prime \prime(n)}(0)+o\left(u^{2}\right), \\
\kappa_{\epsilon_{k \tau}}^{\prime(n)}(u)=\kappa_{\epsilon_{k \tau}}^{(n)}(0)+u \kappa_{\epsilon_{k \tau}}^{\prime \prime(n)}(0)+\frac{u^{2}}{2} \kappa_{\epsilon_{k \tau}}^{(3)(n)}(0)+o\left(u^{2}\right), \\
\kappa_{\epsilon_{k \tau}}^{\prime \prime(n)}(u)=\kappa_{\epsilon_{k \tau}}^{\prime \prime(n)}(0)+u \kappa_{\epsilon_{k \tau}}^{(3)(n)}(0)+\frac{u^{2}}{2} \kappa_{\epsilon_{k \tau}}^{(4)(n)}(0)+o\left(u^{2}\right) .
\end{array}
$$

Using the relationships between the cumulants $K_{j}=\kappa_{\epsilon_{k \tau}}^{(j)(n)}(0)$ and the raw moments of the GARCH innovations, $K_{1}=0, K_{2}=1, K_{3}=M_{3}$ and $K_{4}=M_{4}-3$, we derive the following useful limits:

$$
\begin{aligned}
\lim _{\tau \rightarrow 0} \frac{1}{\tau} \kappa_{\epsilon_{k \tau}}^{(n)}\left(-\sqrt{\tau} \theta_{k \tau}^{(n)}\right) & =\frac{1}{2} \theta_{t}^{2}, \quad \lim _{\tau \rightarrow 0} \frac{1}{\tau} \kappa_{\epsilon_{k \tau}}^{(n)}\left(\sqrt{\tau}\left(\sqrt{h_{k \tau}^{(n)}}-\theta_{k \tau}^{(n)}\right)\right)=\frac{1}{2}\left(\sqrt{h_{t}}-\theta_{t}\right)^{2} . \\
\lim _{\tau \rightarrow 0} \frac{1}{\sqrt{\tau}} \kappa_{\epsilon_{k \tau}}^{(n)}\left(-\sqrt{\tau} \theta_{k \tau}^{(n)}\right) & =-\theta_{t}, \quad \lim _{\tau \rightarrow 0} \kappa_{\epsilon_{k \tau}}^{\prime \prime(n)}\left(-\sqrt{\tau} \theta_{k \tau}^{(n)}\right)=1 .
\end{aligned}
$$

Here, $\theta_{t}$ represents the weak limit of $\theta_{k \tau}^{(n)}$. Taking the limit of the martingale equation (3.7) and using the above results, we get:

$$
\theta_{t}=\nu_{t}^{(1)}=\lambda^{(\epsilon)}+\frac{\frac{1}{2} h_{t}-\kappa_{t}\left(\sqrt{h_{t}}\right)}{\sqrt{h_{t}}} .
$$

After some tedious algebraic manipulations, the drifts coefficients of the bivariate diffu- 
sions under $Q_{\text {ess }}^{(n)}$ are obtained from the following limits:

$$
\begin{aligned}
\lim _{\tau \rightarrow 0} \frac{1}{\tau} \mathrm{E}^{Q_{e s s}^{(n)}}\left[\Delta Y_{k \tau}^{(n)} \mid \mathcal{F}_{(k-1) \tau}^{(n), h_{t}}\right] & =r-\frac{1}{2} h_{t}, \\
\lim _{\tau \rightarrow 0} \frac{1}{\tau} \mathrm{E}^{Q_{e s s}^{(n)}}\left[\Delta h_{(k+1) \tau}^{(n)} \mid \mathcal{F}_{(k-1) \tau}^{(n), h_{t}}\right] & =\omega_{0}-\left(\omega_{1}+\sqrt{\omega_{2}}\left(M_{3}-2 \omega_{3}\right) \nu_{t}^{(1)}\right) h_{t} .
\end{aligned}
$$

In a similar way, the diffusion coefficients are obtained from a Cholesky decomposition of the a symmetric matrix with the following elements:

$$
\begin{aligned}
\lim _{\tau \rightarrow 0} \frac{1}{\tau} \mathrm{E}^{Q_{e s s}^{(n)}}\left[\left(\Delta Y_{k \tau}^{(n)}\right)^{2} \mid \mathcal{F}_{(k-1) \tau}^{(n), h_{t}}\right] & =h_{t}, \\
\lim _{\tau \rightarrow 0} \frac{1}{\tau} \mathrm{E}^{Q_{e s s}^{(n)}}\left[\left(\Delta h_{(k+1) \tau}^{(n)}\right)^{2} \mid \mathcal{F}_{(k-1) \tau}^{(n), h_{t}}\right] & =\omega_{2}\left(M_{4}^{*}-1+4 \omega_{3}^{2}-4 M_{3}^{*} \omega_{3}\right) h_{t}, \\
\lim _{\tau \rightarrow 0} \frac{1}{\tau} \mathrm{E}^{Q_{e s s}^{(n)}}\left[\Delta Y_{k \tau}^{(n)} \Delta h_{(k+1) \tau}^{(n)} \mid \mathcal{F}_{(k-1) \tau}^{(n), h_{t}}\right] & =\sqrt{\omega_{2}}\left(M_{3}^{*}-2 \omega_{3}\right) \sqrt{h_{t}^{3}} .
\end{aligned}
$$

In the above equations, $M_{j}^{*}=\lim _{\tau \rightarrow 0} \mathrm{E}^{Q_{e s s}^{(n)}}\left[\left(\epsilon_{k \tau}^{*(n)}\right)^{j} \mid \mathcal{F}_{(k-1) \tau}^{(n), h_{t}}\right], j=3,4$. The only thing remaining to be showed is that these quantities coincide with the corresponding raw moments under $P^{(n)}$. We first express the cumulant generating function of $\epsilon_{k \tau}^{*(n)}$ under $Q_{\text {ess }}^{(n)}$ :

$$
\kappa_{\epsilon_{k \tau}^{*}}^{*(n)}(u)=-u \frac{\kappa_{\epsilon_{k \tau}}^{\prime(n)}\left(-\sqrt{\tau} \theta_{k \tau}^{(n)}\right)}{\sqrt{\kappa_{\epsilon_{k \tau}}^{\prime \prime(n)}\left(-\sqrt{\tau} \theta_{k \tau}^{(n)}\right)}}-\kappa_{\epsilon_{k \tau}}^{(n)}\left(-\sqrt{\tau} \theta_{k \tau}^{(n)}\right)+\kappa_{\epsilon_{k \tau}}^{(n)}\left(\frac{u}{\sqrt{\kappa_{\epsilon_{k \tau}}^{\prime \prime(n)}\left(-\sqrt{\tau} \theta_{k \tau}^{(n)}\right)}}-\sqrt{\tau} \theta_{k \tau}^{(n)}\right) .
$$

Evaluating the third and fourth derivatives at zero, and using the same useful limit results from the beginning of the proof, we obtain the following cumulant limits under $Q_{\text {ess }}^{(n)}$ :

$$
\begin{aligned}
& K_{3}^{*}=\lim _{\tau \rightarrow 0} \kappa_{\epsilon_{k \tau}^{*}}^{*(n)}(0)=\lim _{\tau \rightarrow 0} \frac{\kappa_{\epsilon_{k \tau}}^{(3)(n)}\left(-\sqrt{\tau} \theta_{k \tau}^{(n)}\right)}{\left(\kappa_{\epsilon_{k \tau}}^{\prime \prime(n)}\left(-\sqrt{\tau} \theta_{k \tau}^{(n)}\right)\right)^{3 / 2}}=K_{3}, \\
& K_{4}^{*}=\lim _{\tau \rightarrow 0} \kappa_{\epsilon_{k \tau}^{*}}^{*(n)}(0)=\lim _{\tau \rightarrow 0} \frac{\kappa_{\epsilon_{k \tau}}^{(4)(n)}\left(-\sqrt{\tau} \theta_{k \tau}^{(n)}\right)}{\left(\kappa_{\epsilon_{k \tau}}^{\prime \prime(n)}\left(-\sqrt{\tau} \theta_{k \tau}^{(n)}\right)\right)^{2}}=K_{4} .
\end{aligned}
$$

Based on the connections between moments and cumulants we thus have $M_{j}^{*}=M_{j}, j=$ 3, 4. Finally, the relations between the Brownian motions under $P$ and $Q$ are obtained by matching the bivariate diffusions dynamics. 